Slavica

bruxellensia

\section{Slavica bruxellensia}

Revue polyphonique de littérature, culture et histoire

slaves

$3 \mid 2009$

Pensée des hommes

\title{
Boris Mouravieff, Écrits sur Ouspensky, Gurdjieff et sur la Tradition ésotérique chrétienne
}

\section{Olivier Santamaria}

\section{OpenEdition \\ Journals}

Édition électronique

URL : http://journals.openedition.org/slavica/212

DOI : $10.4000 /$ slavica. 212

ISSN : 2034-6395

\section{Éditeur}

Université libre de Bruxelles - ULB

\section{Édition imprimée}

Pagination : 103

ISSN : 2031-7654

Référence électronique

Olivier Santamaria, « Boris Mouravieff, Écrits sur Ouspensky, Gurdjieff et sur la Tradition ésotérique chrétienne », Slavica bruxellensia [En ligne], 3 | 2009, mis en ligne le 15 juin 2009, consulté le 22 septembre 2020. URL : http://journals.openedition.org/slavica/212 ; DOI : https://doi.org/10.4000/ slavica. 212

Ce document a été généré automatiquement le 22 septembre 2020.

\section{(i) (9)}

Les contenus de Slavica bruxellensia sont mis à disposition selon les termes de la Licence Creative Commons Attribution - Pas d'Utilisation Commerciale - Pas de Modification 3.0 France. 


\section{Boris Mouravieff, Écrits sur Ouspensky, Gurdjieff et sur la Tradition ésotérique chrétienne}

Olivier Santamaria

\section{RÉFÉRENCE}

Boris Mouravieff, Écrits sur Ouspensky, Gurdjieff et sur la Tradition ésotérique chrétienne, Paris, Dervy, 2008, 342 p. 
La maison Dervy vient de publier, à

l'initiative de l'Association Boris

Mouravieff, un recueil d'une dizaine articles parus entre 1954 et 1961 notamment dans la «Revue Suisse d'Histoire» et dans la revue belge "Synthèses », lesquels étaient devenus à peu près introuvables. Les sujets abordés sont divers: certains articles traitent de théologie politique ( Le problème de l'homme nouveau », «Liberté, égalité, fraternité », «L'Histoire a-t-elle un sens?») ou d'histoire religieuse («Des

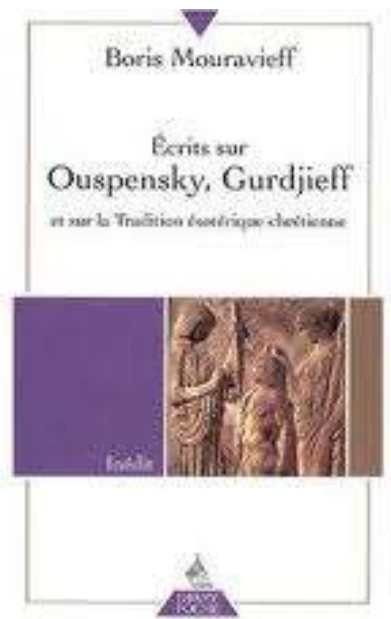
croyances slaves pré-chrétiennes", «Sainte-Sophie de Constantinople »), d'exégèse spirituelle (« Le substantiel et l'Essentiel », «Du "pain quotidien"») ou de figures importantes de l'ésotérisme ("Ouspensky, Gurdjieff et les Fragments d'un Enseignement inconnu », « Additif à l'article "Ouspensky..."»).

De façon très opportune, les éditeurs de ce volume ont également décidé de publier quelques textes parus entre 1961 et 1972 qui étaient réservés jusqu'ici aux membres du Centre d'Etudes Chrétiennes Esotériques (C.E.C.E.), fondé à Genève en 1961 par Mouravieff lui-même et destiné à faire connaître son œuvre et en particulier les trois volumes de Gnôsis - Études et commentaires sur la Tradition ésotérique de l'Orthodoxie orientale. Ces textes sont d'une part des «bulletins d'information » assez courts, proposant aux «étudiants» conseils et exercices spirituels, d'autre part des «Stromates » qui sont des compléments, des applications concrètes, ou des clés de compréhension de la doctrine exposée dans Gnôsis.

Le volume offre donc dans l'ensemble un aspect composite, non seulement parce que les textes ont été rédigés pour des publics différents - puisque inégalement sensibilisés à la pensée de l'auteur - mais encore parce que les articles touchent à des sujets variés. Néanmoins, l'unité est préservée par l'intention fondamentale de Mouravieff, qui est celle de prendre pour point de départ la doctrine spirituelle (la "science ésotérique ») et d'en tirer des conclusions applicables dans tous les domaines du savoir, ainsi que des solutions concrètes et des moyens pour agir sur la politique et sur l'histoire. L'auteur invite donc tant les chercheurs que les hommes d'État à dépasser certains cloisonnements mentaux et à répondre ensemble, sur de nouvelles bases, aux problèmes de l'Humanité. Il s'agit bien, comme l'écrivent Claude Thomas et Jean Poyard dans leur introduction, « de contribuer à la formation "d'hommes nouveaux" capables, selon l'expression de saint Paul, de "renouveler l'esprit de leur intelligence" ». 
INDEX

Index géographique : Russie

Index chronologique : communisme, entre-deux-guerres

Mots-clés : ésotérisme chrétien, orthodoxie

\section{AUTEURS}

\section{OLIVIER SANTAMARIA}

Doctorant à l'Université Libre de Bruxelles (Belgique), aspirant FNRS ; membre du Centre interdisciplinaire d'étude des religions et de la laïcité (CIERL) de l'Université Libre de Bruxelles (Belgique) 